\title{
Identification of Vibrio parahaemolyticus Strains by means of HPLC fingerprinting
}

\author{
Yiling Tian, Yunzhe Zhang, Hui Shi, Wei Zhang \\ Agriculture University of Hebei, Baoding 071001, China \\ email: tougantian@126.com
}

Keywords: Vibrio parahaemolyticus, identification, high performance liquid chromatography

\begin{abstract}
The objective of this study was to identify Vibrio parahaemolyticus by HPLC, a simple, rapid, and reliable HPLC method. For the HPLC methods, an established for the fingerprints of Vibrio parahaemolyticus was performed on Strict extraction and reversed-phase HPLC conditions. An Agilent C18(4. $6 \mathrm{~mm} \times 25 \mathrm{~mm}, 5 \mu \mathrm{m})$ as column and acetonitrile-0.05\% phosphoric acid solution in gradient as mobile phase. The detection wavelength was set at $280 \mathrm{~nm}$ and column temperature was $30^{\circ} \mathrm{C}$. Established HPLC fingerprint was distinct and reproducible. There were 2 characteristic peaks. The HPLC fingerprints of Vibrio parahaemolyticus is characteristic and specific, and has the potential to replace biochemical methods because of its simplicity, rapidity, specificity, and cost-effectiveness. It is apparent from this study that we had developed a new, steady and rapid method for identification of Vibrio parahaemolyticus Strains. For the rapid identification of foodborne pathogens build a technology platform.
\end{abstract}

\section{Introduction}

Vibrio parahaemolyticus is a halophilic bacterium that widely spread in estuarine and coastal marine environments and can be detected in various types of seafood throughout the world. Food poisoning associated with this microorganism is commonly reported[1,2]. Vibrio parahaemolytics infection can cause gastroenteritis in humans, and the illness is most frequently associated with the consumption of raw or undercooked seafood and seafood recontaminated with the bacterium after cooking[3-5].

Biochemical methods is conventional culture methods. Culture is time-consuming, taking several weeks to produce results and is relatively insensitive. Molecular methods is an accurate and rapid method for identifying of Vibrio parahaemolyticus would aid in the prevention of such outbreaks. PCR, has been used to identify the presence of tdh (thermostable direct hemolysin)and/or trh (thermostable direct hemolysin-related hemolysin)genes in Vibrio parahaemolyticus[6,7]. Among the different PCR methods developed in recent years for Vibrio parahaemolyticus identification. Therefore, for disease prevention, after the isolation . The advantages of this application are higher speed and less handling of PCR products such as electrophoretic analysis. However, owing to the expensive systems required, this application is still not very common in laboratories[8].

High performance liquid chromatography (HPLC) is a method to assay ingredients, it is the most common method for identification and quantification. Presently, the research advances of microorganism by HPLC are almostly Mycobacterium genus.

Mycobacterium genus, cannot be identified by conventional biochemical methods. So it analysed by high performance liquid chromatography , developed for disease control and prevention. Over the last decade, HPLC analysis of the mycolic acids has become an accepted method for identification of mycobacteria. In this review, we assess its development and usefulness as an identification technique for microorganism.[9-11]

This paper describes a first attempt to determine Vibrio parahaemolyticus Strains by means of HPLC fingerprinting. The most significant advantage of HPLC fingerprinting is steady and rapid. 


\section{Materials and methods}

\section{Reference Strain.}

For the evaluation of primer specificity, a total of 16 bacterial strains were used.bacterial stains were prepared from six reference stains . The six are purchased from American Type Culture Collection(ATCC), China Center of Industrial Culture Collection(CICC), National Center For Medical Culture Collections(CMCC), Marine Culture Collection of China(MCCC). Vibrio parahaemolyticus (ATCC17802, ATCC27519, CICC21618), Vibrio cholerea(CMCC17005), two Vibrio furnissii (MCCC1D00127, MCCC1H00051) and 10 isolates of Vibrio were obtained from seafood samples purchased at supermarkets were tested using HPLC assay to show specificity. Vibrio spp. were cultured in $100 \mathrm{ml}$ tryptic soya broth culture medium supplemented with $2.5 \%(\mathrm{w} / \mathrm{v}) \mathrm{NaCl}$ and incubated $15 \mathrm{~h}$ at $35-37^{\circ} \mathrm{C}$.

\section{Extraction from culture.}

After incubation at $37^{\circ} \mathrm{C}$ for $15 \mathrm{~h}$. The bacterial suspension was prepared by centrifuged for $20 \mathrm{~min}$ at $4000 \mathrm{r} / \mathrm{min}$. Then washed with $3 \% \mathrm{NaCl}$ of deionized water. The residue was then collected into a clean tapered polypropylene test tube (50 ml capacity) with $30 \mathrm{ml}$ of a methanolic, sonicated for $0.5 \mathrm{~h}$, centrifuged for $10 \mathrm{~min}$ at $4000 \mathrm{r} / \mathrm{min}$ and the eluent was evaporated to dryness under a stream of $\mathrm{N}_{2}$ at $50^{\circ} \mathrm{C}$. The evaporated residue was dissolved in methanol and made up to volume in $1 \mathrm{ml}$. The solutions were filtered through a membrane filter $(0.45 \mu \mathrm{m})$ and then injected into the HPLC.

Before analysis of HPLC, experimental conditions, such as incubation time and concentration for extraction, were kept the same for all strains whenever possible to ensure comparability of the results.

\section{Reversed-phase HPLC conditions.}

The final separation of fingerprinting of Vibrio parahaemolyticus was achieved by reversed-phase HPLC. The column configuration was an Agilent C18 reserved-phase column $(5 \mu \mathrm{m}, 250 \times 4.6 \mathrm{~mm})$. The sample injection volume was $20 \mu$ l. The detection wavelength was set at $280 \mathrm{~nm}$, the flow rate was $1 \mathrm{ml} / \mathrm{min}$ and the column temperature was maintained at $30^{\circ} \mathrm{C}$. The mobile phase consisted of $\mathrm{A}$ (acetonitrile) and B (0.05\% phosphoric acid).

The multi-step gradient elution was as follows:

0-5minutes(5\%A,95\%B),5-20minutes5\%A-65\%A,95\%B-35\%B),20-25minutes(65\%A-80\%A, 35\%B-20\%B),25-27minutes(80\%A-90\%A,20\%B-10\%B),27-40 minutes (90\%A, 10\%B). After each analysis, $5 \%$ mobile phase $A$ was pumped and maintained for $10 \mathrm{~min}$ to re-equilibrate the system for baseline stability.

\section{Results}

Fingerprint analysis of six reference stains.

In the laboratory test performed on strains, a total correct identifications as Vibrio parahaemolyticus on tested and did not produce any false-positive result.

As peaks was isolated from Vibrio parahaemolyticus in our chemistry study, two characteristic peaks were taken as standard substance to perform fingerprint assay. As shown in Fig. 1, according to the

two characteristic peaks of HPLC fingerprint, the retention times were $16.873 \mathrm{~min}, 21.955 \mathrm{~min}$. 


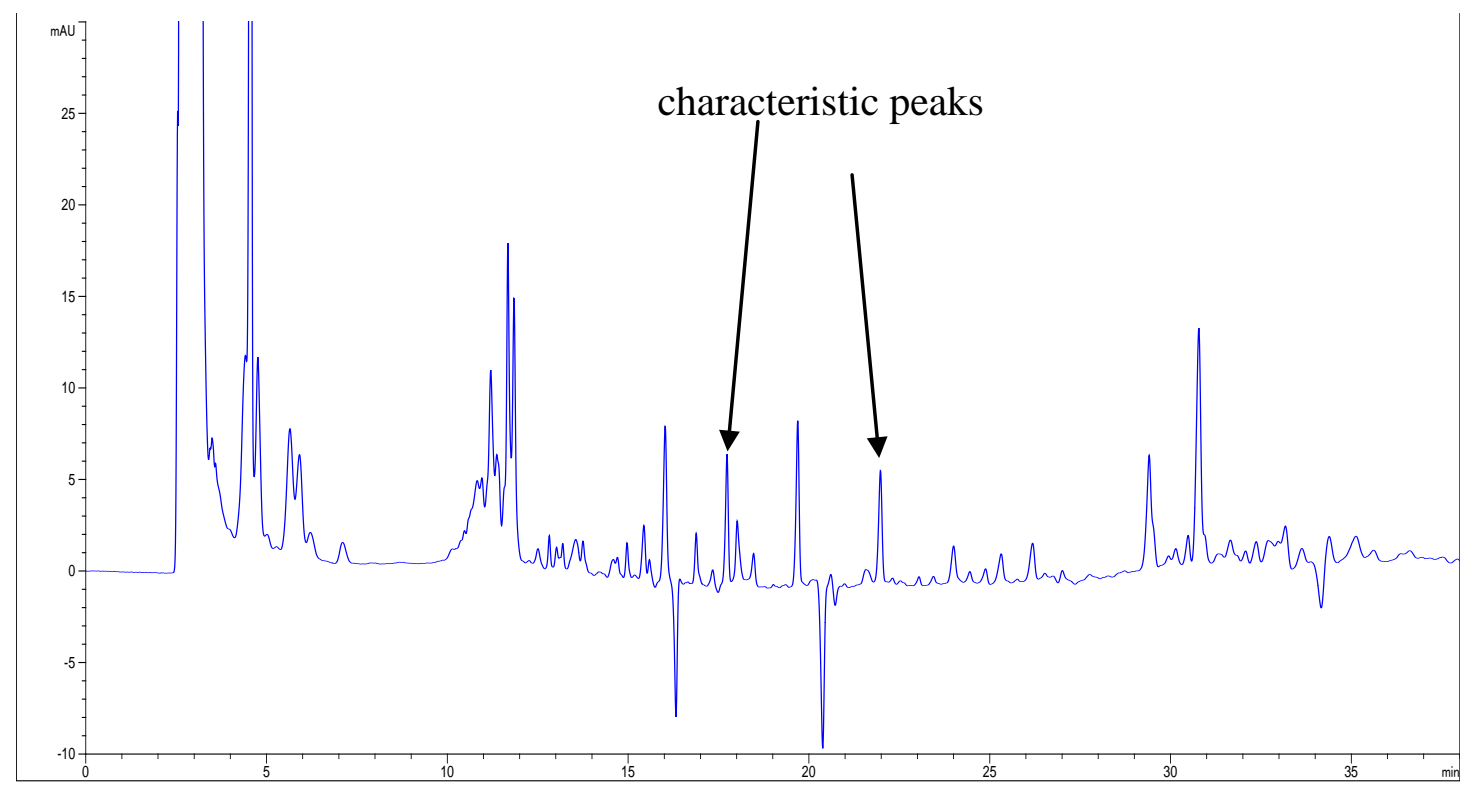

Fig. 1. peaks are separated from Vibrio parahaemolyticus（ATCC17802）.

Representative chromatograms of three Vibrio parahaemolyticus stains (ATCC17802 、 ATCC27519、CICC21618) are show in Fig. 2. HPLC fingerprint of Vibrio parahaemolyticus stains are similar and have the two characteristic peaks. HPLC fingerprinting is steady.

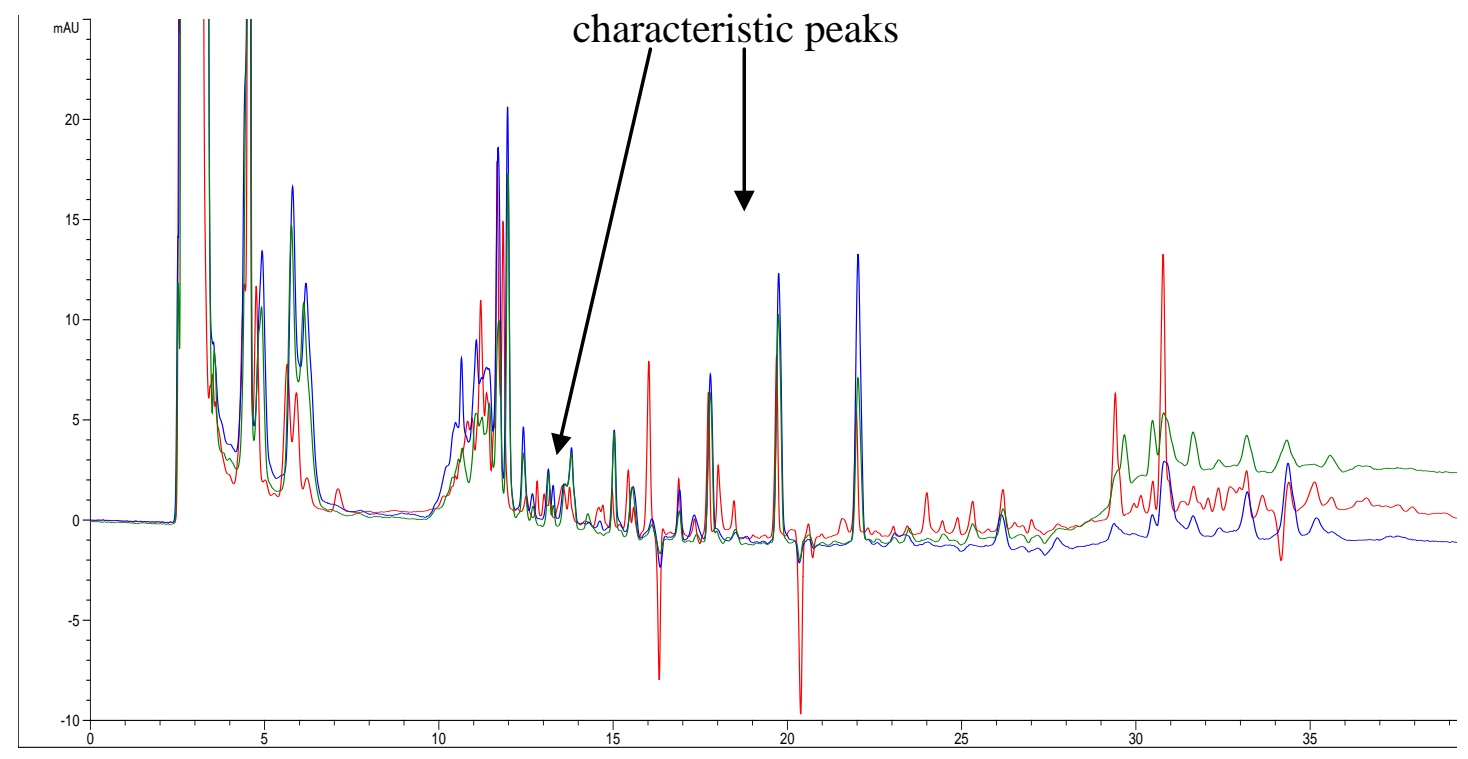

Fig. 2. HPLC fingerprint of Vibrio parahaemolyticus stains(ATCC17802、ATCC27519、 CICC21618).

Fig. 3 shows typical chromatograms of Vibrio parahaemolyticus (ATCC17802) 、 two Vibrio furnissii (MCCC1D00127, MCCC1H00051) and vibrio cholerea (CMCC17005). The specific peaks from Vibrio parahaemolyticus but not from any of the other bacterial species. 


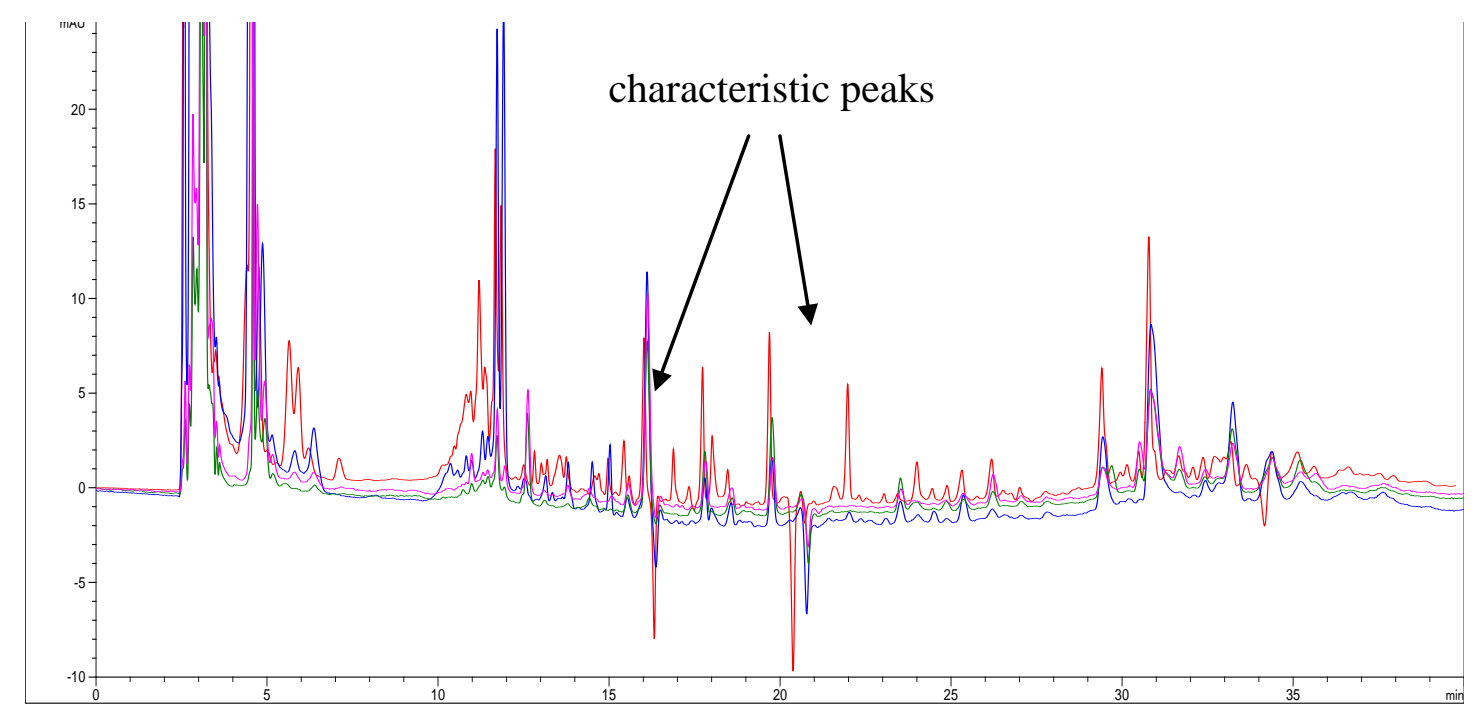

Fig. 3. The red is Vibrio parahaemolyticus (ATCC17802), two Vibrio furnissii (MCCC1D00127, MCCC1H00051) and vibrio cholerea(CMCC17005)

\section{Fingerprint analysis of ten isolates.}

Ten isolates from seafood samples were analysed using the HPLC, compared with biochemical methods. For both HPLC and biochemical methods, four of ten isolates tested positive and the others tested negative. Thus, the concordance between the two assays was $100 \%$. No inhibition of the HPLC assay was identified among the HPLC-assay-negative specimens in this study.

These results demonstrate that it has high specificity for the amplification of Vibrio parahaemolyticus and identified it with high efficiency.

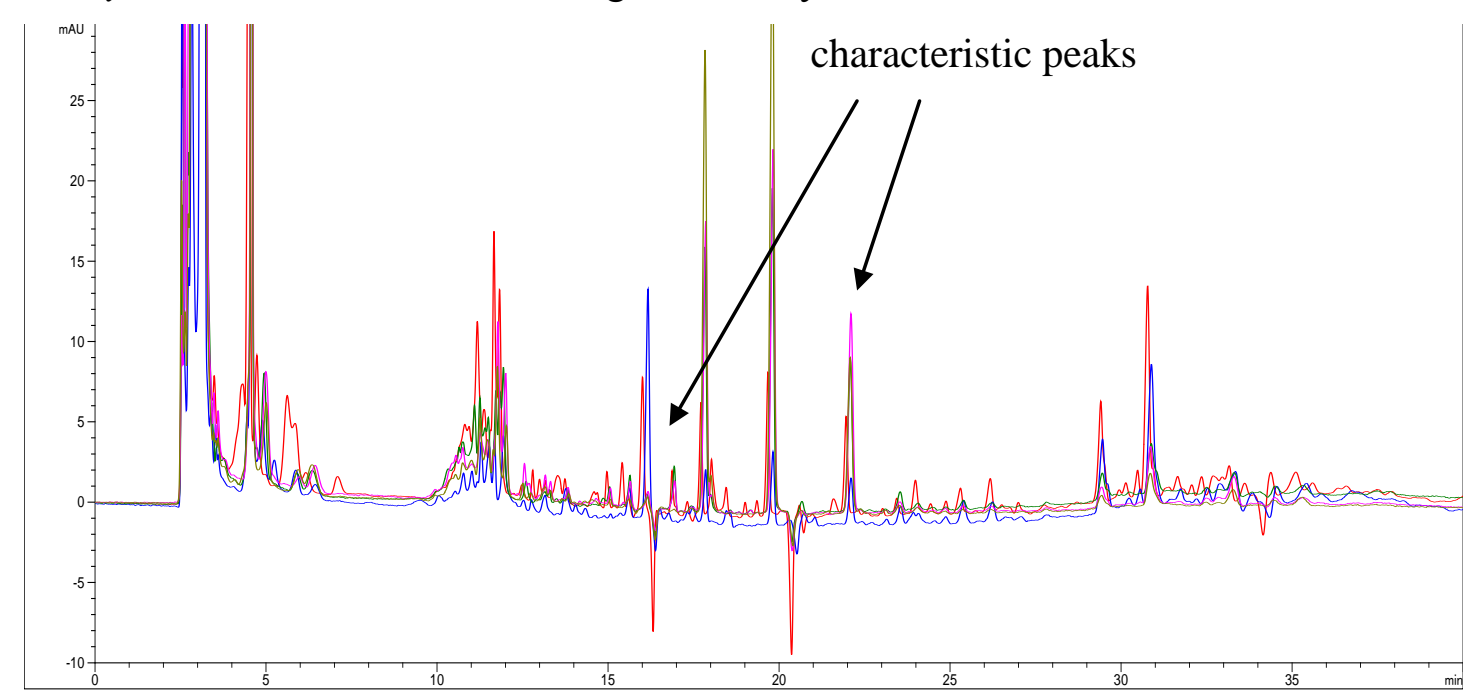

Fig. 4. The red is Vibrio parahaemolyticus (ATCC17802), four of ten isolates tested positive, have characteristic peaks. it could be inferred that it was Vibrio parahaemolyticus. 


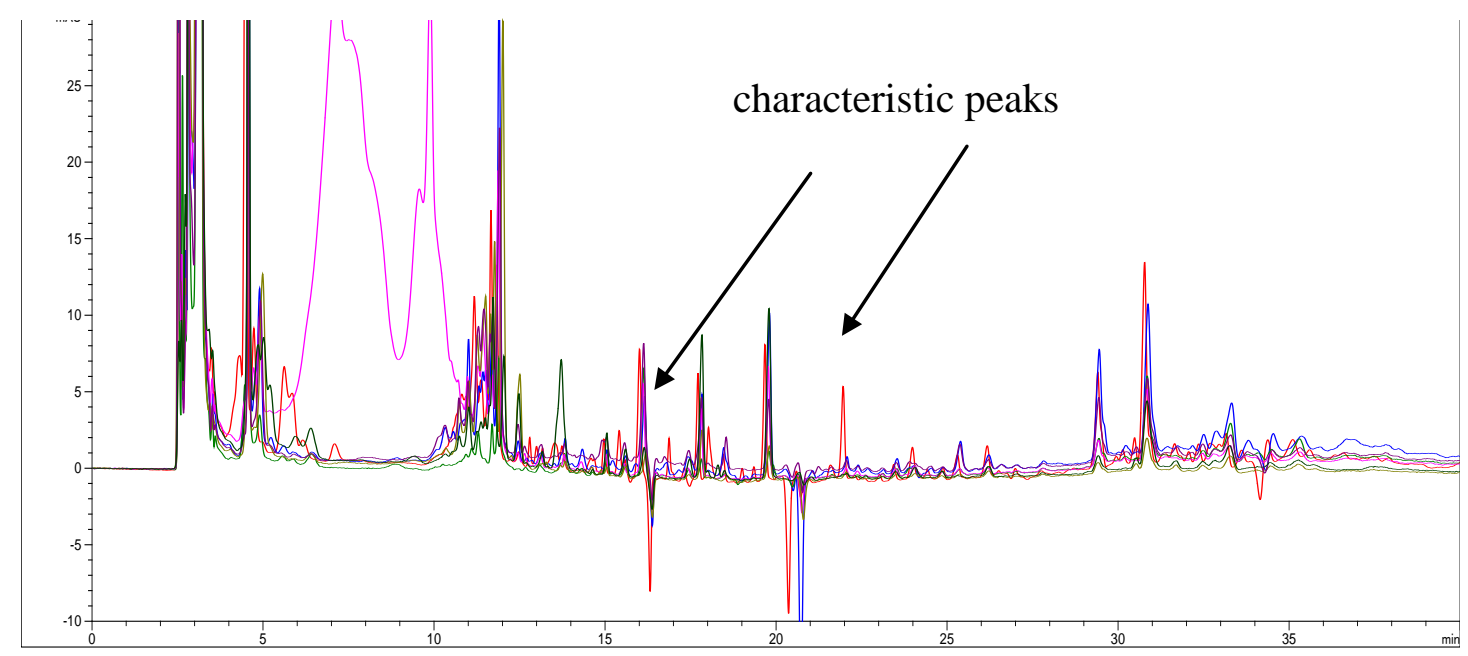

Fig. 5. The red is Vibrio parahaemolyticus (ATCC17802), six of ten isolates tested negative, have not characteristic peaks.

\section{Discussion.}

In order to achieve steady and characteristic extraction, variables involved in the procedure such as incubation time ,solvent and extraction time were optimized.we tested $12,13,14,15,16,17 \mathrm{~h}$ of incubation time ,a series of solvents such as water, methanol and methanol: water (7:3, 5:5, 3:7, v/v) were tested as the extraction. Samples were extracted with methanol for 10, 20, 30, 40,50, 60 min. The best incubation time was 15h.The best solvent was found to be methanol. The ultrasonic treatment procedure was found to be the best extraction method . The best time of ultrasonic was 30 min. Through rigorous processing and liquid conditions, we can get good separation and reproducibility.

Among the tests used, the biochemical methods is time-consuming and relatively insensitive, The bacterial culture test for the isolation and identification of Vibrio parahaemolyticus from food samples after enrichment, plating onto selective agars, sequential subculture and biochemical characteristic test, requires more than one week. Conventional PCR assay requires $4-5 \mathrm{~h}$ for amplification, electrophoresis and stainin[12].The method of HPLC only use less than one day from incubation to inject into the HPLC and the HPLC assay requires for extraction and analysis from Vibrio parahaemolyticus strains less than $2 \mathrm{~h}$. The results of the laboratory study showed a higher sensitivity and a good specificity of the Vibrio parahaemolyticus in comparison with the biochemical methods and PCR.

The results of this comparative test suggest that most of the tested methods are able to correctly identify Vibrio parahaemolyticus strains. This HPLC method was not only able to identify correctly the strains of Vibrio parahaemolyticus tested, but did not produce any false-positive result on the strains belonging to other Vibrio species, in the 16 strains tested. In conclusion, this study showed that, among the tested, the fingerprint of HPLC is a new method and the most efficient for Vibrio identification.

\section{Acknowledgement}

The authors gratefully acknowledge financial support of this study by National Natural Science Foundation of China Projects (31371772).

\section{References}

[1] M.Z. Hayat, A. Kassu, A. Mohammad, M. Yamato, N.A. Bhuiyan, N.G. Balakrish, F. Ota, Microbiol Res 161 (2006) 25.

[2] J. Martinez-Urtaza, A. Lozano-Leon, A. DePaola, M. Ishibashi, K. Shimada, M. Nishibuchi, E. Liebana, J Clin Microbiol 42 (2004) 4672. 
[3] A. Deepanjali, H.S. Kumar, I. Karunasagar, I. Karunasagar, Appl Environ Microbiol 71 (2005) 3575.

[4] G.E. Kaufman, M.L. Myers, C.L. Pass, A.K. Bej, C.A. Kaysner, Lett Appl Microbiol 34 (2002) 155.

[5] P.A. Lang, S. Kaiser, S. Myssina, C. Birka, C. Weinstock, H. Northoff, T. Wieder, F. Lang, S.M. Huber, Cell Microbiol 6 (2004) 391.

[6] F. Raimondi, J.P. Kao, C. Fiorentini, A. Fabbri, G. Donelli, N. Gasparini, A. Rubino, A. Fasano, Infect Immun 68 (2000) 3180.

[7] N. Gonzalez-Escalona, G.M. Blackstone, A. DePaola, Appl Environ Microbiol 72 (2006) 7925.

[8] V. Dileep, H.S. Kumar, Y. Kumar, M. Nishibuchi, I. Karunasagar, I. Karunasagar, Lett Appl Microbiol 36 (2003) 423.

[9] W.R. Butler, J.O. Kilburn, J Clin Microbiol 26 (1988) 50.

[10] W.R. Butler, L.S. Guthertz, Clin Microbiol Rev 14 (2001) 704.

[11] M.V. Makarova, M.A. Krasnova, A.M. Moroz, Zh Mikrobiol Epidemiol Immunobiol (2009) 64.

[12] L. Croci, E. Suffredini, L. Cozzi, L. Toti, D. Ottaviani, C. Pruzzo, P. Serratore, R. Fischetti, E. Goffredo, G. Loffredo, R. Mioni, J Appl Microbiol 102 (2007) 229. 